\title{
Attractive boson and the gas-liquid condensation
}

\author{
Shun-ichiro Koh* \\ Physics Division, Faculty of Education, Kochi University, Akebono-cho, 2-5-1, Kochi 780, Japan \\ (Received 12 January 2001; revised manuscript received 22 May 2001; published 13 September 2001)
}

\begin{abstract}
Gas-liquid condensation (GLC) in the attractive uniform Bose gas is studied by applying the idea of the Yang-Lee zeros to its grand partition function. The followings are proved: (i) When the temperature decreases and the density increases, the GLC occurs prior to the Bose-Einstein condensation (BEC), (ii) an explosive growth of the Bose-statistical coherence to a macroscopic scale occurs simultaneously with the GLC, which are triggered by bosons with a zero momentum, and (iii) the GLC in the Bose gas is not only a condensation in coordinate space, but also in momentum space. On the basis of these results, a comparative study of the BEC and the GLC is developed. Further, we discuss its implication to the trapped atomic gas.
\end{abstract}

DOI: 10.1103/PhysRevB.64.134529

PACS number(s): 64.60.-i, 03.75.Fi, 05.30.Jp, 05.70.Fh

\section{INTRODUCTION}

The study of the relationship between the Bose-Einstein condensation (BEC) and the gas-liquid condensation (GLC) is a long-standing problem. ${ }^{1}$ They are remarkable phenomena of the many-body system at low temperature and high density.

Normally, the GLC is thought to be an essentially different phenomenon from the BEC for the following reasons:

(i) The BEC is regarded as a condensation in momentum space, while the GLC occurs in coordinate space.

(ii) The BEC is attributed to the Bose statistics so that it still occurs without any interaction between particles, while the GLC does not occur without the interactions.

The GLC is a phenomenon not only in the classical gas but also in the quantum gas. An interesting point of the quantum gas is that the reason by which we distinguish the BEC and the GLC is not as obvious as it looks. This problem assumes a quite different aspect in Fermi and Bose statistics.

The attractive fermions form the Cooper pairs at low temperature and high density: a BEC in a general sense. Because of Fermi statistics, however, the fermion still has a large kinetic energy at the zero temperature, and the two fermions experience a strong repulsive force in the short-distance. Hence, although the Cooper pairs form the BEC, Fermi statistics underlying the particle prevents the GLC in general. [We can find a concrete example of this property in the Bardeen-Cooper-Schriefer (BCS) model. As long as the attractive force is increased within the BCS model, the GLC is impossible, a rigorous proof of which was given recently. $\left.{ }^{2}\right]$

At high temperature and low density, the bosons are in a gas state as well as the fermions. With decreasing temperature, however, the bosons lose the kinetic energy. (It is especially obvious in the BEC state.) Further, in contrast with the fermion, the boson does not experience a repulsive force in the short distance. These behaviors create an instability which is characteristic of Bose statistics. When the attractive force acts on the bosons having the small kinetic energy, its influence must be drastic. The compressibility is no longer positive definite, so that the dilute Bose system will collapse into the dense one, leading to the GLC. This is illustrated schematically in Fig. 1(a) (the dotted circle is the zero-point motion).
Conversely, if the GLC occurs prior to the BEC, the density increases locally by the GLC, and an overlapping of the wave function will lead to the BEC as depicted in Fig. 1(b) (the wavy curve is the wave function). These natures suggest that, in the attractive Bose gas, the BEC and the GLC enhance each other. In other words, Bose statistics enhance the $G L C$.

Unfortunately for the low-temperature physics, however, helium 4, our most popular Boson system, is not a good example for this problem. In helium 4, the GLC occurs as a classical phenomenon. (The difference between the transition temperature in ${ }^{4} \mathrm{He}$ and in ${ }^{3} \mathrm{He}$ is small: $T_{c}=4.215 \mathrm{~K}$ in ${ }^{4} \mathrm{He}$ and $3.191 \mathrm{~K}$ in ${ }^{3} \mathrm{He}$ at $1 \mathrm{~atm}$, suggesting that quantum statistics plays a minor role in this GLC.) This well-known but not obvious fact is attributed to the fact that the value of $T_{c}$ is too high for Bose statistics to play a dominant role in this GLC. ${ }^{3}$ Recently, experimental realizations of the BEC in dilute atomic gas changed this academic problem to a realistic one. ${ }^{4}$ The GLC of the ultracold gas has a possibility of elucidating the role of quantum statistics in the GLC clearly. In this paper, we consider this role using the uniform Bose gas, and apply it to the trapped Bose gas.

The instability of the Bose-Einstein condensate with the attractive interaction was already known in the Bogoliubov model as an appearance of an imaginary sign in the velocity of the sound propagating through the condensate, which has been considered to be a kinematical evidence of the GLC. ${ }^{5}$ Behind the kinematics of the many-body system, a thermodynamical reason driving the system to undergo the GLC must exist. This paper studies the GLC in the Bose gas from a viewpoint of the statistical mechanics. ${ }^{6}$ With decreasing temperature and increasing density, the chemical potential $\mu$ of the Bose gas approaches zero from a negative side. Hence the behavior of the grand partition function at the small negative $\mu$ gives us a crucial information on the $\mathrm{BEC}$ and the GLC. A key question is which of the BEC and the GLC occurs first when cooling and compressing the attractive Bose gas.

The scheme of this paper is as follows. Section II describes a perturbation-theoretic derivation of the grand partition function of the attractive uniform Bose gas. Using this result, we prove that (i) when cooling and compressing the attractive Bose gas, the GLC occurs prior to the BEC, and 

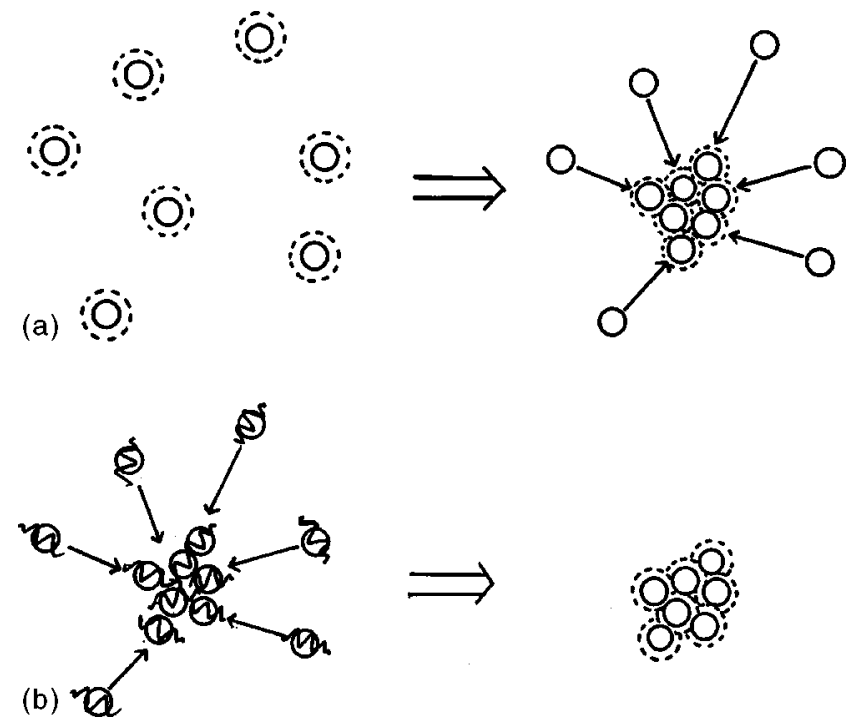

FIG. 1. A schematic view of the role of Bose statistics in the GLC. (a) When the attractive force acts on the bosons with the zero momentum (the dotted circle represents the zero-point motion), it leads to the GLC. (b) When the wave functions of the bosons overlap due to the GLC, it will lead to the BEC.

(ii) the bosons with a zero momentum play a special role in this instability. Section III describes where the GLC occurs in the phase diagram. Section IV deals with an application to the trapped Bose gas. Section V discusses similarities and differences between the BEC and the GLC, and gives a different view on this long-standing problem.

\section{FORMALISM}

The GLC is considered as a singularity in the isothermal pressure vs specific-volume diagram. The pressure $p$ and the density $\rho$ are given by

$$
\begin{gathered}
\frac{p}{k_{B} T}=\lim _{V \rightarrow \infty} \frac{\ln Z_{V}}{V}, \\
\frac{\rho}{k_{B} T}=\lim _{V \rightarrow \infty} \frac{\partial}{\partial \mu}\left(\frac{\ln Z_{V}}{V}\right),
\end{gathered}
$$

where $Z_{V}$ is the grand partition function in the volume $V$, and $\mu$ is a chemical potential. Yang and Lee ${ }^{7}$ proved the following: (i) As $V \rightarrow \infty$ under the constant $N / V, V^{-1} \ln Z_{V}$ approaches a continuous and monotonous increasing function of $\mu$. (ii) If $Z_{V}$ approaches zero at $\mu=\mu_{c}, V^{-1} \ln Z_{V}$ in the $V \rightarrow \infty$ limit is continuous, but $(\partial / \partial \mu) V^{-1} \ln Z_{V}$ is in general discontinuous at $\mu=\mu_{c}$. Hence its isotherm turns out to be discontinuous and not differentiable at the critical specific volume, exhibiting the GLC. ${ }^{8}$

The problem is whether or not the grand partition function $Z_{V}(\mu)=\operatorname{Tr} \exp [-\beta(H-\mu N)]$, which does not seem to be singular at first sight, really shows such a singular behavior. Yang and Lee pointed out that, if zeros of the $Z_{V}$ in the complex $\mu$ plane approach the real $\mu$ axis as $V \rightarrow \infty$ at $\mu_{c}$, such a GLC really occurs.
Complementary to their approach, we can directly regard $Z_{V}(\mu)=0$ at $\mu_{c}$ as an event on the real $\mu$ axis from the beginning. An important point for confirming occurrence or nonoccurrence of the GLC in the cold and dense Bose gas is that one must take into account a large-scale coherence due to Bose statistics in the grand partition function, so that it is beyond the reach of the conventional linked-cluster expansion. If we want to obtain $Z_{V}(\mu)$ by means of the perturbative expansion, we must consider many "unorthodox" diagrams which are not seen in the ordinary linked-cluster expansion. If $Z_{V}(\mu)=0$ occurs in such an expansion at $\mu_{c}$, this means that the gas shows an instability. As a matter of fact, we can obtain only $Z_{V}(\mu)$ with some approximation (we call this a model). If $Z_{V}(\mu)=0$ is confirmed at $\mu$ $=\mu_{c}$, the occurrence of the GLC is proved at least within this approximation. Conversely, if such an expansion does not lead to $Z_{V}(\mu)=0$, the nonoccurrence of the GLC is proved at least within this approximation. In the statistical physics, the microscopic derivation of the GLC on the basis of the grand partition function has been a difficult problem. ${ }^{9}$ If we find a new workable model of the GLC on the physical ground, it will help us to understand the GLC.

Let us consider a spinless Bose gas with a repulsive core represented by $H_{r e}$ and a weak attractive $s$-wave pairing interaction $H_{a t}$ with $g(<0):^{10}$

$$
H=\sum_{p} e_{p} a_{p}^{\dagger} a_{p}+H_{r e}+\frac{g}{V} \sum_{p, p^{\prime}} a_{p}^{\dagger} a_{-p}^{\dagger} a_{-p^{\prime}} a_{p^{\prime}},
$$

where the summation over $p$ in the interaction term is cut off by $p_{h c}$ corresponding to the hard-core radius of the particle. Here, we make the following assumptions: (i) diluteness of the gas allows a contact interaction $g$ as a first approximation, (ii) the slow collision at low temperature allows an assumption of elastic s-wave interaction ${ }^{11}$, and we consider an instability inherent in this gas.

From now, we regard $\Sigma_{p} e_{p} a_{p}^{\dagger} a_{p}+H_{r e}$ as an unperturbed Hamiltonian (kinetic energy $e_{p}$ is replaced by quasiparticle energy $\left.\epsilon_{p}\right)$, and obtain a grand partition function $Z_{V}(\mu)$ $=\operatorname{Tr} \exp [-\beta(H-\mu N)]$ by the perturbation theory with respect to the attractive interaction $H_{a t}$,

$$
\begin{aligned}
Z_{V}(\mu)= & Z_{0} \sum_{n=0}^{\infty} \frac{(-1)^{n}}{n !} \int_{0}^{\beta} d \beta_{1} \cdots \\
& \times \int_{0}^{\beta} d \beta_{n}\left\langle T H_{a t}\left(\beta_{1}\right) \cdots H_{a t}\left(\beta_{n}\right)\right\rangle_{c},
\end{aligned}
$$

where $Z_{0}$ denotes $Z_{V}$ of the unperturbed system, $\beta$ is the inverse temperature, and the cumulant implies the standard definition.

(i) In the single phase, a first approximation of $Z_{V}(\mu)$ is $Z_{0}=\Pi_{p}\left(1-e^{-\beta\left(\epsilon_{p}-\mu\right)}\right)^{-1}$, so that the equation of states has a form as

$$
\frac{p}{k_{B} T}=\frac{g_{5 / 2}\left(e^{\beta \mu}\right)}{\lambda^{3}}-\frac{\ln \left(1-e^{\beta \mu}\right)}{V},
$$




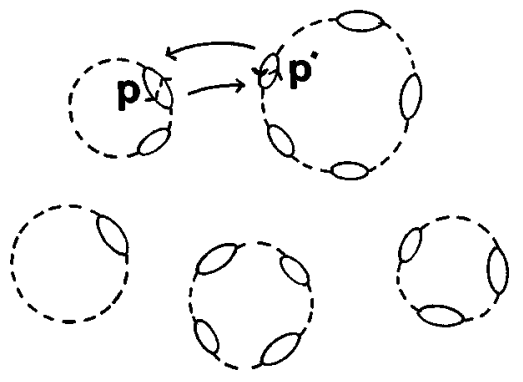

FIG. 2. Some ring diagrams, which are made by connecting the bubbles.

$$
\frac{N}{V}=\frac{g_{3 / 2}\left(e^{\beta \mu}\right)}{\lambda^{3}}+\frac{1}{V} \frac{e^{\beta \mu}}{1-e^{\beta \mu}},
$$

where $g_{a}(x)=\Sigma_{n} x^{n} / n^{a}$ and $\lambda=\left(m k_{B} T / 2 \pi \hbar^{2}\right)^{-1 / 2}$, the thermal wavelength.

A first correction to Eqs. (5) and (6) is made by taking relatively simple diagrams into the expansion of Eq. (4), a typical example of which is a sum of the separated ring diagrams made of the bubble diagram like Fig. 2. (The solid line represents the boson, and the dotted line the attractive interaction. ${ }^{11}$ ) The linked-cluster expansion is a standard method for evaluating such diagrams. Note that such diagrams are only a part of all possible diagrams in Eq. (4). Although such diagrams are very useful for describing the normal properties at high temperature and low density, this $Z_{V}(\mu)$ never can show a singular behavior in the equation of states.

(ii) With decreasing temperature and increasing density, Bose statistics must be dealt with more carefully in Eq. (4). The multiparticle wave function must be wholly symmetric under the exchange of any two particles, which affects the sum of the ring diagrams like Fig. 2 as follows: When two particles $\left(p\right.$ and $\left.p^{\prime}\right)$ belonging to two different bubbles in Fig. 2 have a same momentum $\left(p=p^{\prime}\right)$, a new diagram in which the two particles have been exchanged must be included in the expansion of Eq. (4), ${ }^{12,13}$ which ensures a basic feature of Bose statistics: many identical bosons are likely to occupy the same state $\left(p=p^{\prime}\right)$. As a result, the Fig. 3-type diagram, resulting from many particle exchanges between the bubbles in Fig. 2, becomes important as an exchange correction. In other words, without such diagrams in the expansion, the bosons lose their basic feature in the formalism.

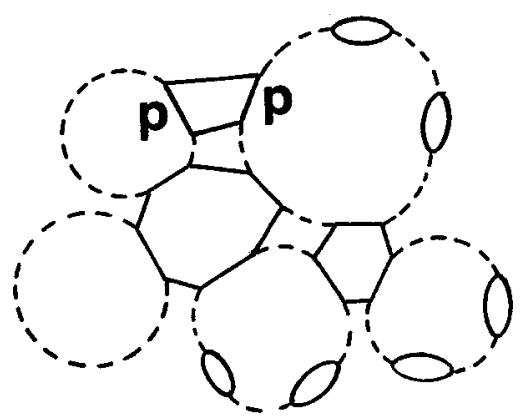

FIG. 3. A joint diagram which is made of the polygons. The ring diagrams in Fig. 2 are united to form this joint diagram.
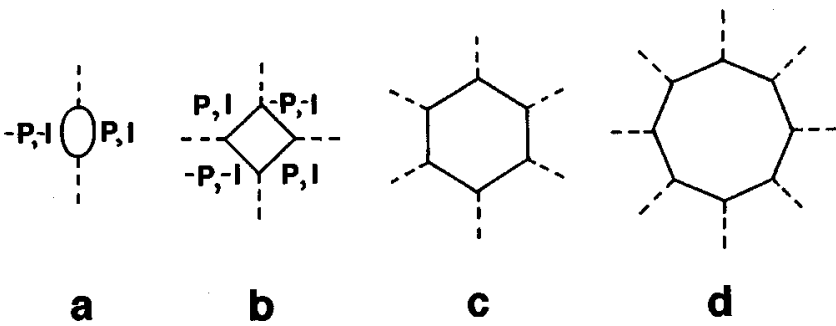

FIG. 4. Four examples of polygons: a bubble $(s=1)$, a square $(s=2)$, a hexagon $(s=3)$, and an octagon $(s=4)$.

In the process of imposing such a permutation symmetry on the sum of ring diagrams, we can generate a new diagram by exchanging particle lines between unlinked ring diagrams: When two particle lines are exchanged between two different bubbles as in Fig. 2, we obtain a square [Fig. 4(b)], which links one unlinked diagram to another as in Fig. 3. Similarly, when three particles are exchanged between three different bubbles, or when two particles are exchanged between a bubble and a square, a hexagon [Fig. 4(c)] is yielded, which links one unlinked diagram to another one. An octagon [Fig. 4(d)] is made by a similar procedure. Such a sequence will continue to a macroscopically large polygon. Starting from $s$ bubbles, a $s$-size polygon ( $s$ gon) is made by $s-1$ times of the exchange of the particle between the bubbles.

In coordinate space, the exchange of Bose particles for ensuring Bose statistics gives us a many-body coherent wave function obeying Bose statistics. Hence the size of the polygons (a number of its sides) in momentum space corresponds to a size of the coherent wave function obeying Bose statistics in coordinate space. With cooling and compressing the system, the coherent wave function will become more important in the cumulant of Eq. (4). Hence in momentum space the perturbative expansion of $Z_{V}(\mu)$ will experience a structural change: Much greater networks of the interaction and particle lines, which are made by connecting the polygons, will appear in the expansion. [More precisely, the complex large diagrams, which are negligible in the single phase, will make major contributions to $Z_{V}(\mu)$.]

An example of such complex diagrams is illustrated in Fig. 3, which is a joint diagram of four types of the polygons. As the order of the perturbation expansion increases, a variety of such complex diagrams increases rapidly. [Figure 3 is only one example of all possibilities which may appear in the expansion of Eq. (4) as $n=15$.] $Z_{V}(\mu)$ which systematically includes such a large-scale coherence due to Bose statistics will have a crucial importance. For obtaining such a $Z_{V}(\mu)$, the bubble diagram is not appropriate for a unit of complex diagrams. Rather, to describe Bose statistics rigorously, we must begin with the polygons, examples of which are listed in Fig. 4.

The proof of this paper consists of two steps: The first step is to obtain a concrete form of $Z_{V}(\mu)$ including the macroscopic-scale coherence. For this purpose, we will apply the method, originated for the attractive Fermi gas by Goudin $^{12}$ and developed by Langer, ${ }^{13}$ to the attractive Bose gas. The second step is to confirm $Z_{V}(\mu)=0$ at a critical 
chemical potential. We describe the first in subsection A, and the second in subsection B. For completeness, we repeat the formalism in Refs. 12 and 13 in a simplified form, and proceed to the Bose gas.

\section{A. Grand partition function $Z_{V}(\mu)$}

To analyze the macroscopic-scale coherence, we think of two levels of the structure in the perturbative expansion of $Z_{V}(\mu)$ : we call an upper one a joint diagram and a lower one a polygon. A convenient way to enumerate the cumulant in Eq. (4) is to associate each factorization of the cumulant with a joint diagram such as Fig. 3, so that $Z_{V}(\mu)$ is a sum of the various types of the joint diagram. Instead of the linkedcluster expansion, we begin with a combinatorial analysis of networks of interaction lines and particle lines in the joint diagram. Accordingly, instead of the bubble, we regard polygons depicted in Fig. 4 as elementary units. All joint diagrams are made of various types of polygons. (The bubble is a simplest polygon [Fig. 4(a)], so that the ring diagram (Fig. 2 ) is incorporated into the category of the joint diagrams as a simplest one.)

\section{Polygons}

As illustrated in Fig. 4, each polygon is composed of $s$ bosons with a common $(p, l)$, and another $s$ bosons with a common $(-p,-l)$, an expression of which is given by

$$
\left(\frac{1}{\left(\epsilon_{p}-\mu\right)+i \frac{\pi l}{\beta}}\right)^{s}\left(\frac{1}{\left(\epsilon_{-p}-\mu\right)-i \frac{\pi l}{\beta}}\right)^{s} .
$$

[In Fig. 4, four examples $s=1,2,3,4$ are depicted. Because the dotted line represents the elastic $s$-wave interaction, we consider only a common $( \pm l, \pm p)$ in each polygon. ${ }^{14} \mathrm{We}$ assume $\left.\epsilon_{p}=\epsilon_{-p}.\right]$

Consider a polygon $K_{s}$ with $2 s$ bosons. We must consider various environments of the polygons in the joint diagram. Since the polygons in general have different $(l, p)$ in the joint diagram, we define a sum of polygons over different $(l, p)$. Further, because an interaction line connects two polygons in the joint diagram, we include $(g / V)^{s}$ in each polygon $K_{s}$ so as to count each interaction only once, so that we define ${ }^{15}$

$$
K_{s}=\frac{1}{V} \sum_{l, p}\left(-\frac{g}{V} \frac{1}{\beta} \frac{1}{\left(\epsilon_{p}-\mu\right)+i \frac{\pi l}{\beta}} \frac{1}{\left(\epsilon_{p}-\mu\right)-i \frac{\pi l}{\beta}}\right)^{s} .
$$

This $K_{s}$ represents a unit diagram more general than the bubble, where $s$ represents the polygon size, thus indicating a coherence size in which Bose statistics is satisfied rigorously. (The bubble corresponds to $K_{1}$.)

\section{Joint diagrams}

Consider a joint diagram in which the polygon $K_{s}$ appears $\nu_{s}$ times, a distribution of which is expressed as $\left\{\nu_{s}\right\}=\left\{\nu_{1}, \nu_{2}, \ldots\right\}$ (for example, in Fig. 3 $\left.\left\{\nu_{s}\right\}=\{6,1,1,1,0, \ldots\}\right)$. The joint diagram including such a

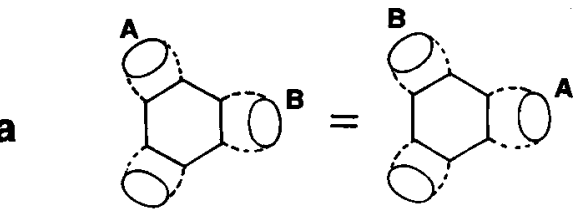

b
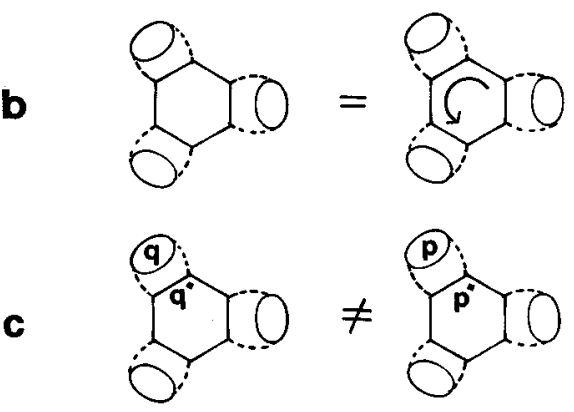

FIG. 5. (a) A permutation of the same type of polygons. (b) A rotation of a polygon. (c) A distribution of $(l, p)$ on the polygons.

polygons has a form $\left(K_{1}\right)^{\nu_{1}}\left(K_{2}\right)^{\nu_{2}} \ldots$ To obtain $Z_{V}(\mu)$, we must sum the joint diagram $\Pi_{s}\left(K_{s}\right)^{\nu_{s}}$ over all possible infinite sets of positive integers including zero $\left\{\nu_{s}\right\}$. This is accomplished for each $s$ (from 1 to $\infty$ ) by summations over $\nu_{s}$ from 0 to $\infty$. When performing the summation, one should keep in mind the following points so as to count each diagram only once:

(i) For each joint diagram, there are $\nu_{s}$ ! ways of rearrangement of $s$-size polygon $K_{s}$, which leaves the joint diagram invariant [Fig. 5(a)]. Thus $\left(K_{s}\right)^{\nu_{s}}$ must be divided by $\nu_{s} !$.

(ii) For each $K_{s}$, there are $2 s$ ways of rotation, which leave the polygon invariant [Fig. 5(b)]. Thus $K_{s}$ must be divided by $2 s$.

(iii) In the joint diagram, there are a number of ways of distributing frequency $l$ and momentum $p$ to each polygon, and the polygons are connected to each other by $n_{a}$ attractive interaction lines as in Fig. 5(c) $\left(n_{a}=6\right)$. This situation can be paraphrased by saying that all interaction lines are distinguishable, having their "individuality." The interaction line is characterized by the frequency and the momentum $(l, p)$ and $\left(l^{\prime}, p^{\prime}\right)$ carried by the particle which enters or emerges at both ends of the line as depicted in Fig. 5(c). This allows $n_{a}$ ! ways of rearrangements which produce different joint diagrams.

With these in mind for summing $\Pi_{s}\left(K_{s}\right)^{\nu_{s}}$, we obtain the following expression of $Z_{V}(\mu)$ :

$$
\frac{Z_{V}(\mu)}{Z_{0}}=\sum_{\left\{\nu_{s}\right\}} n_{a} ! \prod_{s}^{\infty} \frac{1}{\nu_{s} !}\left(\frac{-V K_{s}}{2 s}\right)^{\nu_{s}},
$$

where $\Sigma_{\left\{\nu_{s}\right\}}$ is a summation over infinite sets of positive integers including zero subject to a constraint we explain below. This expansion of $Z_{V}(\mu)$ is based on an idea which is entirely different from that of the ordinary linked-cluster expansion. (The latter is an expansion in powers of the coupling constant, including only a small class of the large-scale coherence. The former is an expansion with respect to the 
size of the coherent wave function obeying Bose statistics, thus including the large-scale coherence systematically.)

As the size of the system approaches infinity (the thermodynamic limit), large polygons appear more frequently in the joint diagram. This means in Eq. (9) that the size of the polygons represented by $\Pi_{s}$ and the number of each type of polygon represented by $\Sigma_{\left\{\nu_{s}\right\}}$ goes to infinity.

\section{Summation over the number of polygons $\nu_{s}$}

Let us consider the number of each type of polygon first. If the sum over $\nu_{s}$ can be carried out independently to $n_{a}$ in Eq. (9), we simply obtain $Z_{V}(\mu) / Z_{0}=n_{a} ! \Pi_{s}^{\infty} \exp \left(-V K_{s} / 2 s\right)$, but in reality the distribution of the polygons and the number of the attractive interaction line are related to each other by $n_{a}=\sum_{s} s \nu_{s}$. This relation is attributed to a fact that $2 s$ interaction lines emerge from each polygon $K_{s}$. To include this constraint in the summation, and to transform $n_{a}$ ! to a simpler form, an identity

$$
n_{a} !=V \int_{0}^{\infty} d t(V t)^{n_{a}} e^{-V t}
$$

is used, and $n_{a}$ in $(V t)^{n_{a}}$ is replaced by $\Sigma_{s} s \nu_{s}$. Using Eq. (10) in Eq. (9), we obtain,

$$
\frac{Z_{V}(\mu)}{Z_{0}}=V \int_{0}^{\infty} d t e^{-V t} \prod_{s}^{\infty} \sum_{\nu_{s}} \frac{1}{\nu_{s} !}\left(\frac{-V K_{s}}{2 s}\right)^{\nu_{s}}(V t)^{s \nu_{s}} .
$$

We can combine $\left(K_{s}\right)^{\nu_{s}}$ with $(V t)^{s \nu_{s}}$, since they have a common form as $x^{s \nu_{s}}$. We define $K_{s}^{\prime}(t)$ in such a way that $1 / V$ in the right-hand side of Eq. (8) is replaced by $t$ as

$$
K_{s}^{\prime}(t)=\frac{1}{V} \sum_{l, p}\left(-t \frac{g}{\beta} \frac{1}{\left(\epsilon_{p}-\mu\right)^{2}+\left(\frac{\pi l}{\beta}\right)^{2}}\right)^{s},
$$

and we rewrite Eq. (11) as

$$
\frac{Z_{V}(\mu)}{Z_{0}}=V \int_{0}^{\infty} d t e^{-V t} \prod_{s}^{\infty} \sum_{\nu_{s}} \frac{1}{\nu_{s} !}\left(\frac{-V K_{s}^{\prime}(t)}{2 s}\right)^{\nu_{s}} .
$$

This allows the individual sums on $\nu_{s}$ to be done. Hence we obtain a parameter representation of $Z_{V}(\mu)$,

$$
\frac{Z_{V}(\mu)}{Z_{0}}=V \int_{0}^{\infty} d t \exp \left(-V t-V \sum_{s} \frac{1}{2 s} K_{s}^{\prime}(t)\right) .
$$

\section{Summation over the size of polygons s: Coherence-size expansion}

Next, let us consider the size of the polygons. In the single phase, the thermodynamic quantities are determined by the small-scale coherence in which only a few particles are participating. This means that $Z_{V}(\mu)$ is well approximated by the simple diagrams like Fig. 2, so that $\nu_{s}$ of only small-size polygons are summed to infinity. For example, the sum of the ring diagram like Fig. 2 is a simplest case in which only the bubble $K_{1}^{\prime}(t)$ has a nonzero value in Eq. (14), so that ${ }^{16}$

$$
\begin{aligned}
Z_{V}(\mu)= & Z_{0} V \int_{0}^{\infty} d t e^{-V t} \prod_{l=0} \prod_{p=0} \\
& \times \exp \left(t \frac{g}{2 \beta} \frac{1}{\left(\epsilon_{p}-\mu\right)^{2}+\left(\frac{\pi l}{\beta}\right)^{2}}\right) .
\end{aligned}
$$

This $Z_{V}(\mu)$ always has a positive value, so that it leads to a continuous and differentiable $p$ and $\rho$ as the isothermal line of the single phase. Even if other types of $K_{s}^{\prime}(t)(s \geqslant 2)$ are added in Eq. (14), we get a qualitatively similar result.

With decreasing temperature and increasing density, however, the contribution of polygons in the exponent of Eq. (14) will gradually change. Since $K_{s}^{\prime}(t)$ is of an order of $t^{s}$, the exponent is a power series of $t, \Sigma C_{s} t^{s}$, being a coherencesize expansion. In view of Eqs. (12) and (14), a ratio of the coefficient $C_{s}$ of $t^{s}$ to that of $t^{s-1}$ is given by

$$
\frac{C_{s}}{C_{s-1}}=-\frac{s-1}{s} \frac{g}{V \beta} \frac{\sum_{l, p}\left[\left(\epsilon_{p}-\mu\right)^{2}+\left(\frac{\pi l}{\beta}\right)^{2}\right]^{-s}}{\sum_{l, p}\left[\left(\epsilon_{p}-\mu\right)^{2}+\left(\frac{\pi l}{\beta}\right)^{2}\right]^{-(s-1)}} .
$$

(Since $t$ in Eq. (14) is a parameter which is renormalized by $V$ as in $e^{-V t}$, we replace $t$ in $K_{s}^{\prime}(t)[\mathrm{Eq} .(12)]$ by $t / V$ for obtaining this ratio.)

Let us estimate $C_{s} / C_{s-1}$ for a large $s$. At $\mu \ll 0$ (high temperature and low density), the numerator and the denominator of Eq. (16) is approximated by $(-\mu)^{-2 s}$ and $(-\mu)^{-2(s-1)}$, respectively, for a small $p$ and $l$. Hence the former is smaller than the latter for a large $s$, leading to $C_{s} / C_{s-1} \ll 1$. This means that the large polygons make a minor contribution in Eq. (14), which validates the approximation like Eq. (15).

As $\mu \rightarrow 0$, however, for a large $s$, a contribution from $l$ $=0$ becomes dominant in the summation in Eq. (16). Hence an asymptotic form of $C_{s} / C_{s-1}$ is given by

$$
\frac{C_{s}}{C_{s-1}} \rightarrow-\frac{g}{V \beta} \sum_{p} \frac{1}{\left(\epsilon_{p}-\mu\right)^{2}}
$$

When cooling and compressing the system, $1 / \beta$ decreases and $1 /\left(\epsilon_{p}-\mu\right)^{2}$ increases. Since $\mu$ approaches zero at the finite temperature (the BEC), the growth of $1 /\left(\epsilon_{p}-\mu\right)^{2}$ for $p=0$ is faster than the decrease of $1 / \beta$, leading to $C_{s} / C_{s-1} \rightarrow \infty$. This implies that, as $\mu \rightarrow 0$, the large polygons made of the $p=0$ Boson becomes remarkably important, so that the size of polygons contributing to Eq. (14) grows explosively to reach a macroscopic scale. (For comparison with the BEC, see Sec. V.)

Hence, for obtaining $Z_{V}(\mu)$ at low temperature and high density, one must sum $K_{s}^{\prime}(t) / s$ over an integer $s$ from 1 to $\infty$ in Eq. (14). With an aid of the identity 


$$
-\sum_{s=1}^{\infty} \frac{(-x)^{s}}{s}=\ln (1+x),
$$

in Eqs. (12) and (14), we obtain the formula derived in Refs. 12 and 13 ,

$$
\begin{aligned}
\frac{Z_{V}(\mu)}{Z_{0}}= & V \int_{0}^{\infty} d t e^{-V t} \prod_{l=0} \prod_{p=0} \\
& \times\left(1+t \frac{g}{\beta} \frac{1}{\left(\epsilon_{p}-\mu\right)^{2}+\left(\frac{\pi l}{\beta}\right)^{2}}\right) .
\end{aligned}
$$

An important point for our purpose is that, following Bose statistics, one must calculate the infinite product with respect to the frequency in Eq. (19) over an even integer $l$ (including zero). By use of the identity

$$
\prod_{n=1}^{\infty}\left(1+\frac{z^{2}}{(2 n)^{2}}\right)=\frac{2}{\pi z} \sinh \frac{\pi z}{2},
$$

in the numerator and the denominator of each factor in Eq. (19), we obtain a final form,

$$
\begin{aligned}
Z_{V}(\mu)= & Z_{0} V \int_{0}^{\infty} d t e^{-V t} \prod_{p=0}\left(1+\frac{g t}{\beta} \frac{1}{\left(\epsilon_{p}-\mu\right)^{2}}\right) \\
& \times\left(\frac{\sinh \beta \sqrt{\left(\epsilon_{p}-\mu\right)^{2}+\frac{g t}{\beta}}}{\sinh \beta\left(\epsilon_{p}-\mu\right)}\right. \\
& \left.\times \frac{\left(\epsilon_{p}-\mu\right)}{\sqrt{\left(\epsilon_{p}-\mu\right)^{2}+\frac{g t}{\beta}}}\right)^{2} .
\end{aligned}
$$

This formula is the concrete form of the grand partition function of the attractive Bose gas including the macroscopicscale coherence. [Equation (21) includes the linked-cluster expansion as a part.]

\section{B. Zero of $Z_{V}(\mu)$}

Our concern is whether or not $Z_{V}(\mu)=0$ occurs in Eq. (21) in the course of cooling and compressing. The fact that the chemical potential approaches zero when cooling and compressing the system is a fundamental property of the boson system, which does not depend on details of the interaction. An important factor for creating the zero in Eq. (21) is

$$
\prod_{p=0}\left(1+\frac{g t}{\beta} \frac{1}{\left(\epsilon_{p}-\mu\right)^{2}}\right)
$$

which comes from $l=0$ contribution in Eq. (19). At high temperature and low density $(\mu \ll 0)$, this product is positive. With decreasing temperature and increasing density, how-

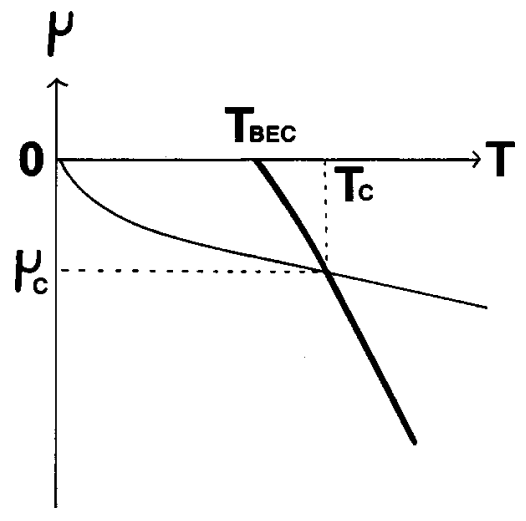

FIG. 6. A schematic graph of $-\sqrt{|g| k_{B} T / V}=\mu(T)$, where the thin solid curve represents $-\sqrt{|g| k_{B} T / V}$ and the thick solid curve $\mu(T)$.

ever, the $\mu$ in the denominator of the second term approaches zero as illustrated in Fig. 6, and the second term in the brackets begins to cancel the first term 1 because of the negative sign $g<0$ (attractive interaction). [Although $1 / \beta$ decreases when cooling, $1 /\left(\epsilon_{p}-\mu\right)^{2}$ increases more rapidly as in Eq. (17).]

Among many factors in the above product, an especially important one is

$$
\left(1+\frac{g t}{\beta} \frac{1}{\mu^{2}}\right)
$$

which comes from $p=0$ boson. Compared with other factors, it decreases most rapidly as the $\mu$ approaches zero from the negative side, because $\left(\epsilon_{p}-\mu\right)^{2}>\mu^{2}$. Finally, for a given $t$, Eq. (23) reaches zero. More precisely, at a critical value $\mu_{c}(<0)$, two integrals, which are obtained by splitting the integrand of Eq. (21) into two parts at this factor, cancels each other. This leads us to conclude that $Z_{V}(\mu)$ becomes zero in the course of cooling and compressing. A concrete estimation of this cancellation will be done in Sec. III. Instead, we discuss the physics behind it in this section.

In summary, the following changes take place as $\mu$ approaches zero.

(i) At $\mu \ll \mu_{c}$, the system is in the gas phase, which is properly described by $Z_{V}(\mu)$ in which the small-scale coherence is dominant $\left(C_{s} / C_{s-1} \ll 1\right)$.

(ii) At $\mu<\mu_{c}, Z_{V}(\mu)$ begins to include the large-scale coherence.

(iii) At $\mu=\mu_{c}(<0)$, the large-scale coherence grows and $Z_{V}(\mu)=0$ occurs. This implies that the density $\rho$ becomes discontinuous in Eq. (2) and that the system really changes to the liquid state in a discontinuous manner. Since $\mu_{c}<0$, we conclude that the GLC occurs prior to the BEC. This suggests that what is necessary for the GLC is not the BEC, but "statistical attraction" due to Bose statistics. (The occupation of the lowest-energy state by a macroscopic number of particles is a too strong condition for the GLC.)

(iv) Normally, the GLC is associated with the metastable state. For any $\mu$ less than $\mu_{c}, Z_{V}(\mu)$ is always positive, but for $\mu_{c} \leqslant \mu, Z_{V}(\mu)=0$ becomes possible. In this sense, $\mu_{c}$ 
gives us a lower limit of the chemical potential for the GLC. For $\mu_{c} \leqslant \mu<0$, if only the system crosses the energy barrier, the GLC must occur prior to the BEC. ${ }^{17}$

One can take the following alternative view on the origin of the instability: The ring diagrams made of the bubbles in Fig. 2 are united to form a joint diagram in Fig. 3 by exchanging the particle lines. This can be viewed as " $a$ condensation of the diagrams" occurring in momentum space. All the joint diagrams can be viewed as a result of such a condensation. This view influences the discussion about the relationship between the BEC and the GLC (see Sec. V).

For the classical gas, the microscopic derivation of the GLC on the basis of the grand partition function has been a difficult problem. ${ }^{9}$ In the attractive Bose gas, however, there is a clear physical origin of the instability due to Bose statistics. Hence a model of the GLC of the attractive Bose gas would be simpler than that of the imperfect classical gas or that of the attractive Fermi gas. This circumstance is a reason why $Z_{V}\left(\mu_{c}\right)=0$ is derived through a relatively simple procedure. The factor coming from $l=0$ in Eq. (21) is a mathematical ingredient of what was predicted by the physical argument on the role of Bose statistics for the GLC.

\section{Role of the zero-momentum boson}

In view of the above argument, one notices that the zeromomentum boson appears twice in the instability mechanism.

(i) As $s \rightarrow \infty$, the ratio of two slightly different-size polygons $C_{s} / C_{s-1}$ increases most rapidly in the zero-momentum boson [Eq. (17)].

(ii) The cancellation of the integrals leading to $Z_{V}(\mu)$ $=0$ occurs in the zero-momentum boson [Eq. (21)].

Both facts indicate a special role of the zero-momentum boson in the GLC. Since the system is not in the BEC phase at $\mu=\mu_{c}$, the number of the zero-momentum boson is not yet a macroscopic one. But it plays a role of trigger for the instability.

Further, one notices also the following:

(iii) For the appearance of the term Eq. (22) in the expression of $Z_{V}(\mu)$, the infinite sum over the polygon size $s$ [Eq. (18)] is crucially important. This fact suggests that the explosive growth of the large-scale coherence is essential for the GLC.

(iv) As a condition of the explosive growth of the coherence in $Z_{V}(\mu)$, one can use $C_{s} / C_{s-1}=1$ for the zeromomentum boson in Eq. (17). As a result, we obtain $-(g / V \beta)\left(1 / \mu^{2}\right)=1$, that is, $\mu=-\sqrt{|g| k_{B} T / V}$. On the other hand, the condition of $Z_{V}(\mu)=0$ in Eq.(21) is more complicated. If two conditions agree completely, this means that $C_{s} / C_{s-1}=1$ and $Z_{V}(\mu)=0$ take place simultaneously due to the zero-momentum bosons. In Sec. III, we will prove this prediction within a first approximation to $Z_{V}(\mu)=0$.

To understand the role of the zero-momentum boson intuitively, we must return to the equation of states. Since the integrand of $Z_{V}(\mu)$ in Eq. (21) has a product form with respect to $p$, in Eqs. (1) and (2) one can approximately distinguish the pressure and the density by the zero-momentum boson from that by other bosons. The total pressure by all the bosons has a positive value at $\mu_{c}$, but the pressure by the zero-momentum boson becomes negative at $\mu_{c}$ because $\ln Z_{V}(\mu)<0$ for $p=0$. Similarly, the total density by all the bosons has a positive value, but the density by the zeromomentum boson discontinuously decreases because $\partial \ln Z_{V} / \partial \mu \rightarrow-\infty$ for $p=0$. A natural interpretation of this fact is that it is the zero-momentum bosons that escape from the dilute gas first, making a liquid droplet, when $\mu$ reaches $\mu_{c}$. This assembly of the zero-momentum bosons with high density is a favorable environment for the BEC. But whether or not this assembly immediately leads to the BEC is a difficult problem (see Sec. V).

\section{Comparison with the Fermi gas}

If we derive $Z_{V}(\mu)$ under Fermi statistics and compare it with Eq. (21), we will obtain a deeper understanding of the role of Bose statistics for the GLC. Let us consider spin one-half fermions having a same form of Hamiltonian as Eq. (3) except that the operators obey Fermi statistics. Its grand partition function has a similar structure as in the boson case. In the Fermi gas, however, one must calculate the infinite product in Eq. (19) over an odd integer $l$. By use of the identity

$$
\prod_{n=1}^{\infty}\left(1+\frac{z^{2}}{(2 n-1)^{2}}\right)=\cosh \frac{\pi z}{2},
$$

in the numerator and the denominator of Eq. (19), we obtain, ${ }^{18}$

$Z_{V}(\mu)$

$$
=Z_{0} V \int_{0}^{\infty} d t e^{-V t} \prod_{p=0}\left(\frac{\cosh \frac{\beta}{2} \sqrt{\left(\epsilon_{p}-\mu\right)^{2}+\frac{|g| t}{\beta}}}{\cosh \frac{\beta}{2}\left(\epsilon_{p}-\mu\right)}\right)^{2} .
$$

This is the grand partition function of the attractive Fermi gas including the large-scale coherence due to Fermi statistics, which is equivalent to the BCS model. ${ }^{12,13}$

Consider a complex $\mu$ plane. $\mu$, which satisfies

$$
\frac{\beta}{2} \sqrt{\left(\epsilon_{p}-\mu\right)^{2}+\frac{|g| t}{\beta}}=i\left(n+\frac{1}{2}\right) \pi,
$$

gives us zeros of $Z(\mu)$. Such a $\mu$ has a form

$$
\mu=\epsilon_{p} \pm i \sqrt{\frac{|g| t}{\beta}+\left(\frac{(2 n+1) \pi}{\beta}\right)^{2}} .
$$

This means that there is no possibility of $Z_{V}(\mu)=0$ for a real $\mu$. Hence, for the attractive Fermi gas equivalent to the BCS model, even if the large-scale coherence is taken into account, the GLC is impossible (see Ref. 2). This conclusion supports the physical argument in Sec. I that Fermi statistics prevents the GLC, and that quantum statistics plays an important role for the GLC of the quantum gas. 


\section{GAS-LIQUID CONDENSATION IN THE PHASE DIAGRAM}

\section{A. Critical chemical potential}

Let us obtain $\mu_{c}$ in a case of the weakly attractive interaction. We approximate the temperature dependence of $\mu$ by that of the free Bose gas (a thick solid curve in Fig. 6), and use the well-known formula of the free Bose gas above $T_{B E C}$, which is derived from Eq. (6) as ${ }^{19}$

$$
\mu(T)=-\left(\frac{g_{3 / 2}(1)}{2 \sqrt{\pi}}\right)^{2} k_{B} T_{B E C}\left[\left(\frac{T}{T_{B E C}}\right)^{3 / 2}-1\right]^{2},
$$

where $\mu(T)$ depends also on the number density $n(=N / V)$ through $T_{B E C}$ as

$$
k_{B} T_{B E C}=\frac{2 \pi \hbar^{2}}{m}\left(\frac{n}{g_{3 / 2}(1)}\right)^{2 / 3} .
$$

When we know $\mu_{c}$, the transition temperature $T_{c}$ of the GLC is defined as $\mu\left(T_{c}\right)=\mu_{c}$ for a given density. As the system approaches $T_{c}$ from above temperature, the Fig. 3-type diagrams become important in $Z_{V}(\mu)$. When it reaches $T_{c}$, the large joint diagram leads to $Z_{V}(\mu)=0$.

Since Eq. (21) has a form of infinite product, it is difficult to estimate its exact value. To obtain a first approximation of $\mu_{c}$, we consider only the most important factor $\left[1+(g t / \beta)\left(1 / \mu^{2}\right)\right]$ in the integrand of Eq. (21), and simplifies $Z_{V}\left(\mu_{c}\right)=0$ as follows:

$$
\int_{0}^{\infty} d t e^{-V t}\left(1+k_{B} T_{c} \frac{g t}{\mu_{c}^{2}}\right)=0 .
$$

[We numerically confirm that an inclusion of the $p \neq 0$ components to Eq. (30) does not change the result so much.] From Eq. (30), we get $\mu_{c} \cong-\sqrt{|g| k_{B} T_{c} / V}$ as a first approximation (a thin solid curve in Fig. 6).

This condition of $\mu_{c}$ is exactly a condition of $C_{s} / C_{s-1}$ $=1$ in Eq. (17) for the zero momentum boson: $\mu=$ $-\sqrt{|g| k_{B} T / V}$. At least within the approximation for $Z_{V}(\mu)$ $=0$ such as Eq. (30), the explosive growth of the coherence $\left(C_{s} / C_{s-1}=1\right)$ and the GLC $\left[Z_{V}(\mu)=0\right]$ take place simultaneously (for its implication, see Sec. V).

The $\mu_{c}$ and the $T_{c}$ are determined as a point of intersection of the thick solid and the thin solid curve in Fig. 6, being a solution of an equation: $-\sqrt{|g| k_{B} T_{c} / V} \cong \mu\left(T_{c}\right)$. Substituting this equation into $T=T_{c}$ in Eq. (28), we get

$$
\mu_{c}=-\left(\frac{g_{3 / 2}(1)}{2 \sqrt{\pi}}\right)^{2} k_{B} T_{B E C}\left[\left(\frac{\mu_{c}^{2}}{\mid \frac{|g|}{V} k_{B} T_{B E C}}\right)^{3 / 2}-1\right]^{2},
$$

and solve $\mu_{c}$ for the small $g$ as

$$
\mu_{c} \cong-\left(\frac{2 \sqrt{\pi} k_{B} T_{B E C}}{g_{3 / 2}(1)}\right)^{2 / 5}\left(\frac{|g|}{V}\right)^{3 / 5} .
$$

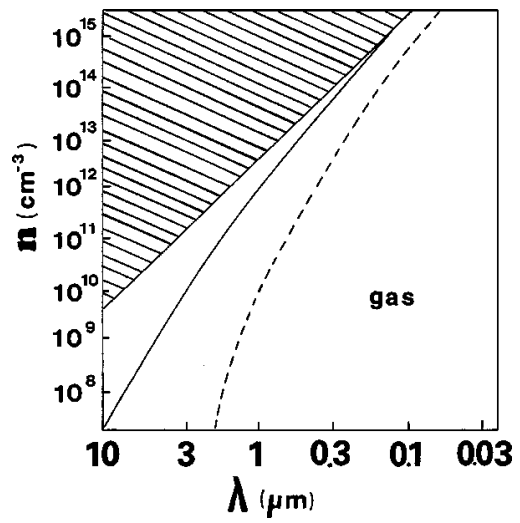

FIG. 7. $(n, \lambda)$ phase diagram of the attractive bosons, where $n$ is a number density and $\lambda=\left(m k_{B} T / 2 \pi \hbar^{2}\right)^{-1 / 2}$. In this figure, the horizontal axis can be viewed just like the temperature axis [in the case of $\mathrm{Rb}$ atom: $T \mathrm{nK}=30 \lambda^{-2} \mu \mathrm{m}$ ]. A solid curve is a GLC line of the $\mathrm{Rb}$ atom defined by $n \lambda^{3}=g_{3 / 2}\left(e^{\beta \mu_{c}(n)}\right)$ with $\mu_{c}(n)$ [Eq. (33)] for $g / V=-5 \mathrm{nK}$, and a dotted curve for $g / V=-5 \mu \mathrm{K}$. Shaded area is the BEC phase of the free Bose gas defined by $n \lambda^{3}$ $\geqslant g_{3 / 2}(1)$.

Equation (32) is a critical value of the chemical potential for the GLC of the weakly attractive Bose gas, showing that a threshold value for the GLC does not exist in the strength of the attractive force: An arbitrary small attractive force is sufficient for the GLC to occur before the chemical potential reaches zero.

\section{B. Condition of the GLC}

Next, let us obtain a condition of this GLC in the phase diagram. For the BEC of the free Bose gas, we have a wellknown condition: $n \lambda^{3}=g_{3 / 2}(1)$, which is derived from Eq. (6). This condition indicates the positions of $(n, \lambda)$ in the phase diagram at which $Z_{V}(\mu) \rightarrow \infty$ because of $\mu=0$.

We modify this formula in such a way as to indicate the position of $(n, \lambda)$ at which $Z_{V}(\mu) \rightarrow 0$ because of $\mu=\mu_{c}$. Accordingly, we replace $\mu=0$ in $n \lambda^{3}=g_{3 / 2}\left(1=e^{0}\right)$ by $\mu_{c}$ such that $n \lambda^{3}=g_{3 / 2}\left(e^{\beta \mu_{c}}\right)$, which determines the density and the temperature at which the GLC occurs. In comparing the two conditions, one notices that, because $e^{\beta \mu_{c}}<1$ and $g_{3 / 2}(x)$ is a monotonic increasing function, one obtains $n_{c}$ $<n_{B E C}$ for a constant $T$, and $T_{c}>T_{B E C}$ for a constant $n$. In the BEC of the free Bose gas, $n \lambda^{3}=g_{3 / 2}(1)$ is a universal line in the $(n, \lambda)$ phase diagram. (All bosons are plotted on this line.) For the GLC, however, a position of $n \lambda^{3}$ $=g_{3 / 2}\left(e^{\beta \mu_{c}}\right)$ in the phase diagram depends on the mass of the boson through the $\mu_{c}$ as

$$
\mu_{c}(n)=-\left(\frac{\hbar}{\sqrt{\pi} m}\right)^{2 / 5} \frac{n^{4 / 15}}{\left[g_{3 / 2}(1)\right]^{2 / 3}}\left(\frac{|g|}{V}\right)^{3 / 5} .
$$

Figure 7 is the $(n, \lambda)$ phase diagram, showing a solid and a dotted curve for $n \lambda^{3}=g_{3 / 2}\left(e^{\beta \mu_{c}(n)}\right)$ (GLC line). The solid curve represents the GLC line of the Rb atom with $\mu_{c}$ [Eq. (33)] for $g / V=-5 \mathrm{nK}$, and the dotted curve represents that for $g / V=-5 \mu \mathrm{K}$. The shaded area represents the BEC phase of the free Bose gas: $n \lambda^{3} \geqslant g_{3 / 2}(1)$. In this figure, the hori- 
zontal axis representing $\lambda=\left(m k_{B} T / 2 \pi \hbar^{2}\right)^{-1 / 2}$ can be viewed just like the temperature axis. [In $\mathrm{Rb}$ atom, $\lambda$ is related to the temperature by $T \mathrm{nK}=30 \lambda^{-2} \mu \mathrm{m}$.]

We note the following features of Fig. 7:

(i) Compared with the BEC region, the GLC lines appear on higher-temperature and lower-density side. Hence, when we start from the ordinary condition, decreasing the temperature and increasing the density of the system, the GLC occurs prior to the BEC.

(ii) Under the same strength of the attractive force, the $\mathrm{BEC}$ region and the GLC line come apart at lower temperature.

(iii) As the strength of the attractive interaction $g$ increases, the position of the GLC line in the phase diagram moves to the higher temperature and lower density region, a more accessible environment. Note that the GLC is very sensitive to $g / V$. It seems natural since the GLC is a strongly cooperative phenomenon.

\section{TRAPPED BOSE GAS}

Let us explore an application to the trapped Bose gas. As discussed in Sec. I, the ultracold trapped Bose gas has a possibility of proving the role of Bose statistics for the GLC. The trapped system, however, has its own properties which complicate the argument developed so far in the uniform system.

The trapped atomic gas is the boson system in the spherical harmonic potential

$$
U(r)=\frac{1}{2} U_{0}\left(\frac{r}{R}\right)^{2},
$$

where $r^{2}=x^{2}+y^{2}+z^{2}$ and $R$ is a range parameter of the potential. The energy level is specified by an integer $M$ $\left(=m_{x}+m_{y}+m_{z}\right.$, a sum of the quantum number) as $e_{M}$ $=\hbar \omega_{0}(M+1)$ where $\omega_{0}=\sqrt{U_{0} /\left(R^{2} m\right)}$. Under this potential, we consider a Hamiltonian of the spinless Bose gas,

$$
H=\sum_{M} e_{M} a_{M}^{\dagger} a_{M}+H_{r e}+\frac{g}{V} \sum_{M, M^{\prime}} a_{M}^{\dagger} a_{-M^{\prime}}^{\dagger} a_{-M^{\prime}} a_{M^{\prime}},
$$

with $g<0$. As in Sec. II, we consider $\Sigma_{M} e_{M} a_{M}^{\dagger} a_{M}+H_{r e}$ as an unperturbed Hamiltonian ( $e_{M}$ is replaced by $\epsilon_{M}$ ), and we consider only the elastic $s$-wave interaction.

This confined system does not have the thermodynamic limit in a strict sense. As $V \rightarrow \infty$, the size of the potential [ $R$ in Eq. (34)] increases, which weakens the potential. As the confinement becomes weaker, the gas near the bottom of the potential approaches the uniform gas in the infinite space, losing the characteristics of the trapped system. (The limit of $N \rightarrow \infty$ under a constant $N \omega_{0}^{3}$ does not maintain the characteristics of the trapped system, which is another expression of $N / V=$ constant for the infinite system existing behind it.) Hence the thermodynamic limit of the trapped gas is impossible not only in a practical sense, but also in principle. We must regard the size of the trapped gas in the experiment as an intermediate scale before approaching the limit. This finite system cannot show mathematical singularities which are established only in the thermodynamic limit. But the trapped gas locally realizes the ultralow temperature and high density, which are impossible in the corresponding uniform gas for technical reasons. Hence it shows macroscopic changes seemingly similar to the phase transition of the uniform gas. (This expectation is supported by some computer simulations of the finite-size systems.) The BEC-like phenomenon is an example of such phenomenon, suggesting that we can expect a GLC-like phenomenon as well. When we focus on such properties, we can study the trapped gas using the grand partition function of the corresponding infinite system. ${ }^{20}$

This infinite system has a unique density of states which reflects the shape of the potential. The lowest energy state which is localized in the bottom of the potential has a relatively large volume in the phase space, compared with the $p=0$ state of the uniform gas: Under a constant $M=m_{x}$ $+m_{y}+m_{z}$, a possible number of states is

$$
\sum_{i=0}^{M}(M+1-i)=\frac{(M+1)(M+2)}{2} .
$$

In the $V \rightarrow \infty$ limit, the number of states can be approximated by $M^{2} / 2$. In the single phase, $Z_{V}(\mu)$ is approximated by $Z_{0}$ $=\Pi_{M}\left(1-e^{-\beta\left(\epsilon_{M}-\mu\right)}\right)^{-M^{2} / 2}$. Hence the equation of states is given by

$$
\begin{gathered}
\frac{p}{k_{B} T}=\frac{g_{4}\left(e^{\beta\left(\mu-\hbar \omega_{0}\right)}\right)}{\lambda_{t}^{3}}-\frac{\ln \left(1-e^{\beta\left(\mu-\hbar \omega_{0}\right)}\right)}{R^{3}}, \\
n=\frac{g_{3}\left(e^{\beta\left(\mu-\hbar \omega_{0}\right)}\right)}{\lambda_{t}^{3}}+\frac{1}{R^{3}} \frac{e^{\beta\left(\mu-\hbar \omega_{0}\right)}}{1-e^{\beta\left(\mu-\hbar \omega_{0}\right)}},
\end{gathered}
$$

where $n=N / R^{3}$ the number density, $\lambda_{t}$ $=\left[m\left(k_{B} T\right)^{2} / \sqrt[3]{2 \Gamma(3)} U_{0} \hbar^{2}\right]^{-1 / 2}$ the thermal wavelength of the trapped gas, and $V=R^{3} \cdot{ }^{20}$

$Z_{V}(\mu)$ in Eq. (21) is revised using a different density of states, so that

$$
\begin{aligned}
Z_{V}(\mu)= & Z_{0} V \int_{0}^{\infty} d t e^{-V t} \prod_{M=0}\left(1+\frac{g t}{\beta} \frac{1}{\left(\epsilon_{M}-\mu\right)^{2}}\right) \\
& \times\left(\frac{\sinh \beta \sqrt{\left(\epsilon_{M}-\mu\right)^{2}+\frac{g t}{\beta}}}{\sinh \beta\left(\epsilon_{M}-\mu\right)}\right. \\
& \left.\times \frac{\left(\epsilon_{M}-\mu\right)}{\sqrt{\left(\epsilon_{M}-\mu\right)^{2}+\frac{g t}{\beta}}}\right)^{M^{2}} .
\end{aligned}
$$

A zero of this $Z_{V}(\mu)$ occurs by a similar mechanism as in Eq. (21). At $\mu=\mu_{c}$, the explosive growth of the large diagram made of $M=0$ boson in $Z_{V}(\mu)\left(C_{s} / C_{s-1}=1\right)$ takes place simultaneously with $Z_{V}(\mu)=0$, signaling the GLC. 
The bosons on the bottom of the potential, the number of which is not yet a macroscopic one at $\mu_{c}$, play a role of a trigger for the instability. ${ }^{21}$

In the trapped system, a formula corresponding to Eq. (28) has a form such as

$$
\mu(T)=\hbar \omega_{0}-\left(\frac{g_{3}(0)}{K}\right) k_{B} T_{B E C}\left[\left(\frac{T}{T_{B E C}}\right)^{3}-1\right],
$$

where

$$
K=\int_{0}^{\infty} d x \frac{x^{2}}{(\sinh x)^{2}},
$$

and the BEC-like transition temperature is defined by ${ }^{20}$

$$
k_{B} T_{B E C}=\frac{\hbar}{\zeta(3)^{1 / 3}} \sqrt{\frac{U_{0} n}{m}} .
$$

Using a similar approximation as in Eq. (30), we get a condition of $\mu_{c}$ from Eq. (39) as $\mu_{c} \cong \hbar \omega_{0}-\sqrt{|g| k_{B} T_{c} / V}$. Hence the $T_{c}$ is obtained by $\hbar \omega_{0}-\sqrt{|g| k_{B} T_{c} / V}=\mu\left(T_{c}\right)$. Substituting this equation to $T=T_{c}$ in Eq. (40), we get a critical value of the chemical potential for the GLC as

$$
\mu_{c} \cong \hbar \omega_{0}-\frac{K\left(k_{B} T_{B E C}\right)^{2 / 5}}{g_{3}(0)}\left(\frac{|g|}{V}\right)^{3 / 5} .
$$

In analogy with $n \lambda^{3}=g_{3 / 2}\left(e^{\beta \mu_{c}}\right)$ in the uniform gas, one obtains from Eq. (38) a condition of the instability for the trapped gas as $n \lambda_{t}^{3}=g_{3}\left(e^{\beta\left[\mu_{c}(n)-\hbar \omega_{0}\right]}\right)$. Using this formula, we can expect a seemingly similar phase diagram to Fig. 7. (The details of the GLC in the trapped Bose gas, including numerical estimation of $n$ and $\lambda_{t}$, will be given in a future paper.)

The BEC-like state with the attractive interaction was observed in trapped ${ }^{7} \mathrm{Li}$ gas as a metastable state. ${ }^{22}$ (At first sight, this contradicts the conclusion of this paper. The metastability at $\mu_{c}<\mu$ prevents the GLC, which complicates the situation.) A peculiar feature of the atomic gas is that we can control the sign of the interaction between the atoms using so-called "Feshbach resonance." Using this technique, the condensate over a wide range of interaction strength is realized. By a sudden switching of the interaction from the repulsive to the attractive one, a collapse of the BEC gas to a high density spot was predicted. Recently such a phenomenon was really observed in ${ }^{85} \mathrm{Rb}^{23}$ When such an experiment is performed with an enough number of atoms to realize the thermal equilibrium, and when a wide region of the normal phase is searched for the occurrence of the GLC, this experiment will provide us with a method of finding a GLClike line of the trapped gas, which corresponds to the GLC line of the uniform Bose gas in Fig. 7.

In the experimentally observed BEC-like state with the attractive interaction, there exists a possibility that some well-known properties of the superfluid, which are believed to be due to the presence of the repulsive interaction, would be altered. But it can happen only within a short lifetime of the metastable state. There is a more drastic catastrophe behind it.

\section{DISCUSSION}

(i) The idea that the GLC is a condensation in coordinate space while the BEC is a condensation in momentum space is questionable. Such a question has been raised by many people in the past. In view of the result of this paper, the GLC of the attractive Bose gas is a phenomenon not only in coordinate space but also in momentum space. We can see this answer in the structural change of the perturbative expansion (from Fig. 2 to Fig. 3), that is, the condensation of the diagrams in momentum space. This view on the GLC is probably true also in the classical gas, though its proof is more difficult than that of the Bose gas. Because of Bose statistics, it becomes possible to understand the GLC simply not only in coordinate space but also in momentum space.

(ii) The explosive growth of the Bose-statistical coherence in the GLC seen in Eq. (16) reminds us of the famous picture of the BEC by Matsubara ${ }^{24}$ and by Feynman. ${ }^{25}$

Feynman expressed the grand partition function $Z_{V}(\mu)$ $=\Sigma_{N} e^{-\mu N} \int d z\left\langle z\left|e^{-\beta H}\right| z\right\rangle$ in terms of the path integral on the imaginary time axis $(u=i t / \hbar)$ from 0 to $\beta$ as follows:

$$
\begin{aligned}
& \left\langle z\left|e^{-\beta H}\right| z\right\rangle \\
& \quad=\int_{t r} \exp \left\{-\int_{0}^{\beta}\left[\frac{m}{2 \hbar^{2}}\left(\frac{d x}{d u}\right)^{2}+V[x(u)]\right] d u\right\} \mathcal{D} x(u),
\end{aligned}
$$

where $x(u)$ denotes "coordinate" of the particle, and $\mathcal{D} x(u)$ denotes a path integral with $t r$ taken on all trajectories forming a circle such that $x(0)=z$ and $x(\beta)=z$.

When this formulation is applied to $N$-boson system, (i) the path of a particle $x(u)$ is replaced by the entire set of paths of $N$ bosons $x_{i}(u)$, and (ii) whereas the initial coordinates are $x_{i}(0)=z_{i}$, the final coordinates need not be the same, but may be some permutations of these. Thus the circle in Eq. (44) is replaced by the permutation circles, each of which is visualized by a "polygon" made of lines joining atomic centers. Hence $Z_{V}(\mu)$ is expressed in terms of the path integral over the polygons.

Note that this polygon has a slightly different meaning from that in the GLC. (i) The polygon in the BEC is a realization of the permutation symmetry essentially in the kinetic energy, in which the interaction is taken into account only through the effective mass. Hence this polygon has no interaction line. It is appropriate to consider the path integral over the polygons as an advanced treatment of $Z_{0}$ in Eq. (4). On the other hand, the polygon in the GLC, from which the interaction line emerges at each vertex, is a realization of the permutation symmetry in the interaction term. (ii) While the polygon in the BEC is defined in coordinate space, the polygon in the GLC is originally defined in momentum space. (iii) The size of the polygon in the BEC can have any positive integers, while the size of the polygon in the GLC has only even positive integers because of the pairing-interaction approximation in Eq. (3). In spite of these differences, however, both polygons reflect the size of the wave function in which Bose statistics is satisfied rigorously in coordinate space. 
At high temperature, the contribution of large polygons to Eq. (44) is small. But as the temperature falls, it suddenly becomes important. The explosive growth of the large polygons in Eq. (44), which results in the macroscopic occupation of the lowest-energy state, is a definition of the BEC. These "motions" of particles in the partition function must not be considered as a real description of what the atoms are doing, but as a formal description of the expression. But the true behavior of the atoms may have some analogy to it. In this sense, this formulation provided us with a realistic picture of the BEC in coordinate space, which had been regarded as a phenomenon in momentum space.

In the GLC as well, we show that the explosive growth of the polygon occurs in the grand partition function, which results in the drastic change of the spatial distribution of the system through Yang-Lee zeros. In contrast with the BEC, the argument on the GLC develops in a reverse direction: The GLC has been regarded as a phenomenon in coordinate space, but the polygon picture of the GLC provides us with a realistic picture of the GLC in momentum space. In this sense, we can find a parallelism in the BEC and the GLC.

Our conclusion that, when cooling and compressing the attractive Bose gas, the GLC occurs prior to the BEC, implies that, in the attractive Bose gas, the remarkable change of the permutation symmetry of the state occurs in two steps, first driven by the attractive-interaction energy, and second by the kinetic energy.

Considering similarities and differences between these two explosive growths of coherence will lead us to a deeper understanding of the relation between the BEC and the GLC.

(iii) In the attractive Bose gas, a possibility of the boson pair has been explored by many people in analogy with the Cooper pair. ${ }^{26}$ In view of the result of this paper, however, the attractive force responsible for such a boson pair must have a property such that there is no residual attractive interaction between the boson pairs. When such a residual force is there, the GLC must occur instead of the boson-pair formation. Forbidding the residual interaction between the boson pair, however, should impose a somewhat artificial constraint on the nature of the attractive interaction between the bosons.

(iv) This paper deals with only an initial stage of the instability. Once the Bose gas changes to the liquid droplet, the repulsive core due to $H_{r e}$ plays a dominant role. Accordingly, the ladder-type diagrams must be taken into account in the perturbative expansion of $Z_{V}(\mu)$ as well. (When the bosons are in a gas state with high density at high temperature, the situation is similar.) This makes Fig. 7 a more complex phase diagram: The critical point $\left(n_{c}, \lambda_{c}\right)$ will appear on the GLC line, and the GLC line below the $\left(n_{c}, \lambda_{c}\right)$ in Fig. 7 must be replaced by the gas-liquid coexisting region. An explicit inclusion of the repulsive force in $Z_{V}(\mu)$ will reveal a competition between the repulsive core and the attractive interaction of bosons, and leads us to understand a more complex nature of the later stage of this instability. An extension of the model along this line is also necessary to understand the unanswered question of whether the liquid droplet made of the zero-momentum boson immediately undergoes the BEC. The later stage of this instability is an open problem.

\section{ACKNOWLEDGMENTS}

The author thanks K. Miyake, Y. Tsue, H. Okamoto, Y. Nagaoka, and K. Burnett and his group for valuable discussion.
*Email address: koh@cc.kochi-u.ac.jp

${ }^{1}$ B. Kahn and G. E. Uhlenbeck, Physica (Amsterdam) 5, 399 (1938).

${ }^{2}$ S. Koh, Phys. Lett. A 229, 59 (1997).

${ }^{3}$ This value of $T_{c}$ is lower than the value predicted by the law of corresponding states, but it is not low enough for all aspects of Bose statistics to appear.

${ }^{4}$ For a review, see A. S. Parkins and D. F. Walls, Phys. Rep. 303, 1 (1998); F. Dalfovo, S. Giorgini, L. P. Pitaevskii, and S. Stringari, Rev. Mod. Phys. 71, 463 (1999).

${ }^{5}$ N. N. Bogoliubov, J. Phys. (USSR) 11, 23 (1947). Recently, for the trapped atomic gas with the attractive interaction, a stability analysis of the Gross-Pitaevskii equation is made by many papers, which can be traced back to this famous model.

${ }^{6}$ In various systems ranging from the nuclear matter to the trapped Bose gas, the Maxwell's equal-area law has been used for insisting on occurrence of the GLC. But the "kink" in the pressurespecific volume curve is attributed to an implicit unphysical assumption of the homogeneity of the system, and it can never be obtained from the exact partition function. In this sense, it is not appropriate to call them statistical mechanical theories. [See, for example, T. Hill, Statistical Mechanics (Dover, New York, 1987)].

${ }^{7}$ C. N. Yang and T. D. Lee, Phys. Rev. B 87, 404 (1952); T. D. Lee and C. N. Yang, ibid. 87, 410 (1952). Although they used the fugacity as a basic variable, we use $\mu$ in this paper.

${ }^{8}$ In contrast to the GLC, the BEC is defined by a pole of $Z_{V}(\mu)$ (at $\mu=0$ for the free Bose gas). Because of the logarithmic function in Eqs. (1) and (2), the pole produces a similar shape in the isothermal curve in comparing with the case of the zero of $Z_{V}(\mu)$. In contrast to the GLC, however, the isotherm of the free Bose gas is not only continuous but also differentiable at the critical volume. (In the repulsive Bose gas, it is continuous but not differentiable.) See, for example, K. Huang, Statistical Mechanics (Wiley, New York, 1987).

${ }^{9}$ J. E. Mayer and M. G. Mayer, Statistical Mechanics (Wiley, New York, 1946).

${ }^{10}$ Pairing interaction is a good approximation of the general attractive interaction, and it simplifies later calculations.

${ }^{11}$ Since the gas is assumed to be dilute, the ladder-type processes are not considered (see Sec. 5).

${ }^{12}$ M. Gaudin, Nucl. Phys. A 20, 513 (1960).

${ }^{13}$ J. S. Langer, Phys. Rev. A 134, 553 (1964). It was proved that the joint diagram like Fig. 3, which is necessary for Fermi statistics, is essential to reproduce the superconductivity in the framework of the standard perturbation theory. We call the "star" in Langers' paper the polygon in this paper.

${ }^{14} \mathrm{~A}$ correct volume dependence of the macroscopic quantities can 
be obtained only after inelastic interactions are properly calculated for $Z_{V}(\mu)$. But we prefer an approximate form of $Z_{V}(\mu)$ representing the instability simply.

${ }^{15}$ In contrast with $K_{s}$ of the Fermi gas in Refs. 12 and 13, a minus sign due to Fermi statistics must not appear in $K_{s}$ of the Bose gas, Eq. (8). But because of a different sign definition of $g$ in this paper to that in Refs. 12 and 13, Eq. (8) has a similar form to $K_{s}$ in these papers.

${ }^{16}$ The product over $p$ in Eq. (15) implies that over not only the magnitude but also the direction of $p$.

${ }^{17}$ When the GLC occurs in the metastable state at $\mu_{c} \leqslant \mu<0$, bosons with nonzero momentum plays a role of the trigger for $Z_{V}(\mu)=0$.

${ }^{18}$ In Eq. (5) of Ref. 2, the volume $V$ in front of the integral was left out, and it must be corrected.

${ }^{19}$ For example, A. L. Fetter and J. D. Walecka, Quantum Theory of
Many-Particle Systems (McGraw-Hill, New York, 1971).

${ }^{20}$ W. J. Mullin, J. Low Temp. Phys. 106, 615 (1997), and references therein.

${ }^{21}$ The quantum fluctuation caused by the confinement within a bottom of the harmonic potential acts as a repulsive interaction between the bosons, which may give rise to a complex nature in the attractive force.

${ }^{22}$ C. C. Bradley, C. A. Sackett, J. J. Tollett, and R. G. Hulet, Phys. Rev. Lett. 75, 1687 (1995)

${ }^{23}$ S. L. Cornish, N. R. Claussen, J. L. Roberts, E. A. Cornell, and C. E. Wieman, Phys. Rev. Lett. 85, 1795 (2000).

${ }^{24}$ T. Matsubara, Prog. Theor. Phys. 6, 714 (1951).

${ }^{25}$ R. P. Feynman, Phys. Rev. 90, 1116 (1953); 91, 1291 (1953).

${ }^{26}$ P. Nozières and D. Saint James, J. Phys. (France) 43, 1133 (1982), and references therein. 